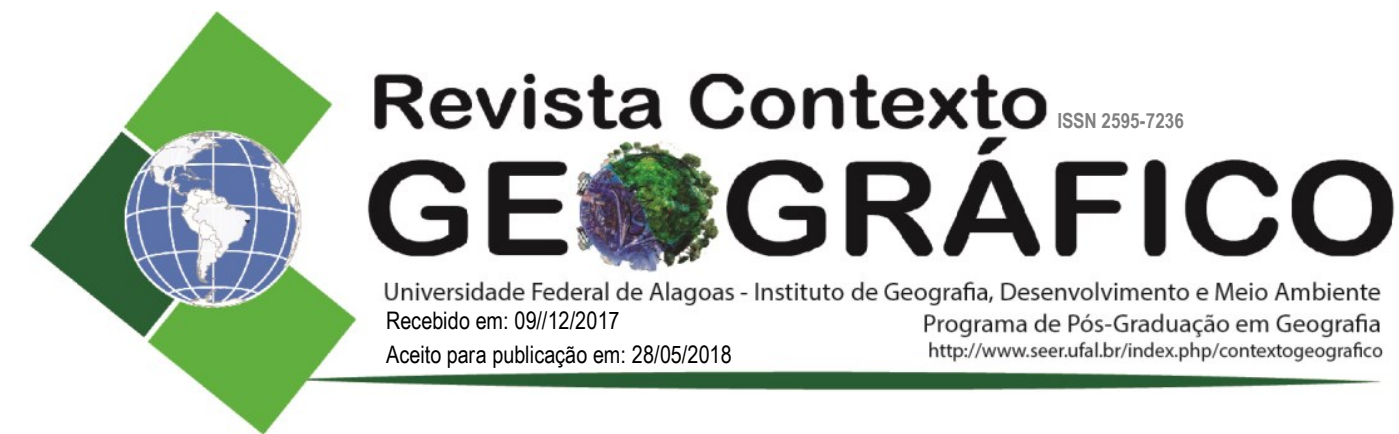

\title{
REFLEXÕES SOBRE DESIGUALDADES REGIONAIS REFERENTES AO USO DA INTERNET NO TERRITÓRIO BRASILEIRO
}

\author{
Jane Roberta de Assis Barbosa \\ Universidade Federal do Rio Grande do Norte, Departamento de Geografia, Natal, RN, Brasil \\ janerabarbosa@gmail.com
}

\begin{abstract}
RESUMO - Nos últimos anos, o acesso a tecnologias digitais no território brasileiro passou por um processo de expansão alcançando novos usuários. O objetivo do artigo é discutir especificamente o uso da internet no território brasileiro, para o que serão estudadas duas variáveis. Primeiramente, o acesso a internet usando telefones celulares. Em segundo lugar, o funcionamento dos telecentros, que se configuram como uma política pública voltada à inclusão digital. A análise dos dados obtidos nos sítios eletrônicos da Agência Nacional de Telecomunicações (Anatel), Ministério da Ciência, Tecnologia, Inovações e Comunicações (MCTIC) e do Instituto Brasileiro de Geografia e Estatística (IBGE), demonstram que persistem desigualdades regionais concernentes ao uso da internet. Estas disparidades mostram uma concentração em subespaços específicos, ressaltando a importância de considerar o uso da Internet como uma categoria para compreender as Dinâmicas Territoriais no Brasil.
\end{abstract}

Palavras-chave: Desigualdade Socioespacial; Dinâmicas Territoriais; Brasil.

\section{CONSIDERATIONS ON REGIONAL INEQUALITIES CONCERNING INTERNET USE IN BRAZILIAN TERRITORY}

\begin{abstract}
In recent years the access to digital technologies in Brazilian territory has expanded considerably reaching new users. The present work aims to address specifically the internet use in Brazil, focusing in two selected variables. Firstly, Web access by means of smartphones, analyzed in the period of 2011 to 2015 . Secondly, telecenter functioning, which is categorized as a public policy directed for digital inclusion. The analysis of database obtained from public Brazilian agencies, such as National Agency of Telecommunications (Anatel), Ministry of Science, Technology, Innovations and Communications (MCTIC) and Brazilian Institute of Geography and Statistics (IBGE), proves that persist regional inequalities related to the use of internet. These disparities show the existence of a concentration in specific sub-spaces which illustrate the importance of considering Internet Use as category for analyzing dynamics related to Brazilian territory.
\end{abstract}

Keywords: Socio-spatial inequalities; Territorial Dynamics; Brazil.

\section{O USO DOS CELULARES E A CAPILARIDADE DA INTERNET NO TERRITÓRIO BRASILEIRO}

Nos dias atuais, computadores e demais objetos técnicos modernos permeiam o cotidiano da sociedade capitalista, onde o processo de inovação das tecnologias de informação e comunicação tornou-se irreversível e se expande seletivamente. O smartphone tem se tornado um complemento ao computador de mesa e notebook nas tarefas relacionadas ao trabalho, serviços, lazer e acadêmicas, uma vez que pelo celular também se pode fazer uso de aplicativos de navegação virtual, e escolher a melhor rota para chegar a um determinado destino. O emprego das novas tecnologias, possibilita uma nova forma de vivenciar as cidades. Todavia, é preciso refletir sobre a desigualdade no acesso às inovações tecnológicas presentes em algumas cidades dos países do Norte e do Sul. A cidade atual caracteriza-se pela profusão de possibilidades desiguais de vivências por meio do uso de tecnologias 
de informação e comunicação que tornam o modo de vida urbano mais complexo e dinâmico, dada à rapidez com a qual se podem acessar informações que permitem circular pelas ruas e fazer uso de equipamentos urbanos de uso coletivo e serviços dispostos à população. Townsend (2014), discute o emprego da tecnologia inteligente nas cidades sob duas perspectivas. A primeira, como produto de investimentos do Estado. A segunda, relacionada ao uso de smartphones que promovem através do acesso à internet, a utilização de aplicativos e redes sociais, que entre outras coisas, possibilita que o cidadão possa exercer influência na gestão das cidades.

Os objetos técnicos modernos, conforme permite refletir Latour (1994), tronaram-se imprescindíveis ao cotidiano dos citadinos, por permitir a formação de híbridos de humanos e não-humanos. A produção desses híbridos têm ressignificado por completo o modo de vida urbano, pois as cidades são por excelência, espaços de produção de conhecimentos (STORPER e VENABLES, 2005), onde as novidades de cada período histórico se instalam com maior expressividade.

A globalização apoia-se em um denso sistema técnico-científico-informacional, o qual cria e transforma as redes geográficas, bem como possibilita que a atuação de grandes empresas, mas não apenas delas, esteja interconectada por uma "mallha" de fixos e fluxos (SANTOS, 2008), permitindo o comando à distância e em tempo real. Por meio da internet, se favorece a dinâmica das empresas e do mercado financeiro, pois o seu uso envolve um conjunto variado de fixos (cabos, fibras ópticas, computadores etc) e fluxos (informação) característicos da atual Divisão Internacional do Trabalho (DIT). Ela ressignifica também as relações sociais, bem como novos modos de produção e trabalho (LÉVY, 1997).

Gras (1993), diz que "a cada época, os objetos ocupam lugares centrais no esquema de pensamento humano e orientam evoluções". Seguindo esse raciocínio, pode-se então refletir sobre o papel das Tecnologias de Informação e Comunicação (TICs), mais notadamente a internet e seu aparato tecnológico, que no período histórico atual, contribui para o estabelecimento do fenômeno denominado por Castells (1999), de sociedade em rede. Para Castells (2003), o estudo da internet pode ser realizado a partir de três perspectivas: infraestrutura, usuários e produção dos equipamentos, enfatizando aspectos relacionados à localização, produção de conteúdos e informação, bem como da economia que se produz a partir dela. Nesse artigo, o enfoque está na segunda perspectiva abordada pelo referido autor.

De acordo com o Suplemento de Tecnologias de Informação e Comunicação (TIC) da Pesquisa Nacional por Amostra de Domicílios (Pnad) 2014 do IBGE, o uso do telefone celular para acessar a internet ultrapassou o do computador. A maior proporção desse uso foi registrada no Nordeste, com $92,5 \%$ dos domicílios com o celular como meio de acesso à internet. A pesquisa revelou ainda que mais da metade dos 67 milhões de domicílios brasileiros passaram a ter acesso à internet em 2014 (54,9\%). Além disso, os dados mostram que 77, $9 \%$ dos entrevistados entre 10 anos ou mais, possuem telefone celular de uso pessoal. O uso do celular abrange todas as classes sociais, gêneros e nível de instrução, mas quando se considera o contexto nacional são as mulheres que detém o maior percentual de aparelhos (78,6\%). Desse modo ratifica-se a importância da reflexão feita por Lévy (1997), para o qual as telecomunicações são responsáveis por ampliar as possibilidades de comunicação e transmissão de informações.

Observa-se, no entanto, que nas regiões Sudeste e Sul, são os homens que detém a maior quantidade de telefones celulares, cujo percentual é de $82,2 \%$ e $82,5 \%$ respectivamente. No que concerne ao nível de instrução, é o segmento da população com ensino superior incompleto e superior completo que mais se destaca. Os dados da Pnad (2014), mostram que no Brasil 97,2\% da população de 10 anos ou mais com ensino superior incompleto possui telefone celular de uso pessoal, já aqueles com ensino superior completo correspondem a 96,3\%. Ressalte-se que na Região Centro-Oeste encontra-se o maior percentual da população sem instrução com telefone celular de uso pessoal $(57,4 \%)$.

A Figura 1 mostra a evolução temporal no número de acessos à internet no Brasil através do uso dos telefones celulares de uso pessoal. $\mathrm{O}$ maior percentual de acessos concentra-se no Sudeste brasileiro 
(73,6\%), sobretudo em estados como São Paulo, Minas Gerais, Rio de Janeiro, além destes, abrange estados do Sul como Paraná e Rio Grande do Sul e Sudeste brasileiro, a exemplo de Goiás e o Distrito Federal, configurando um subespaço de centralidade econômica e financeira do território nacional denominado por Santos e Silveira (2006) de Região Concentrada. Em comparação a outros subespaços do território brasileiro, a partir da Figura 1 é possível afirmar que a Região Concentrada é aquela de maior densidade no acesso à internet, e a Região Norte, representada nesse artigo pelos estados de Roraima e Amapá, é mais rarefeita.

Figura 1. Evolução do acesso à internet por telefone celular por unidade da federação (2011-2016)

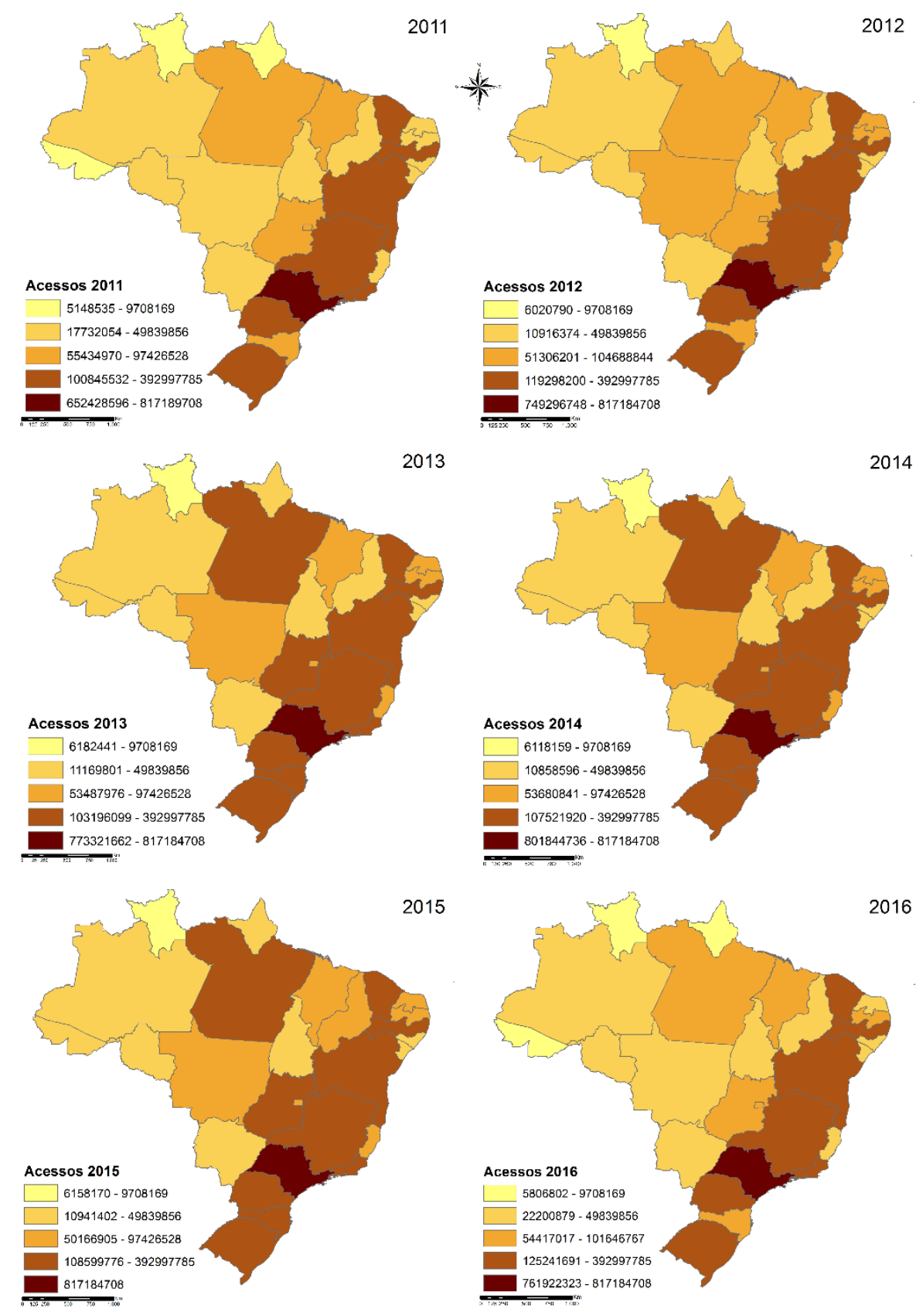

Fonte: Anatel, 2016

Autores: D. Paz \& J. Barbosa 
Tendo em vista que a internet possui um papel de destaque na dinâmica social e econômica do território brasileiro (OLIVEIRA, 2016; ALVES, 2013), embora seu uso ainda não alcance toda a população, pergunta-se: como tem se dado a ação do Estado no sentido de disseminar o seu acesso à população, buscando promover através de políticas públicas a inclusão digital?

Para esse artigo utiliza-se o conceito de inclusão digital a partir de Fuser (2011, p. 20), que é “" ...] a oferta pura e simples de equipamento de informática e conexão à internet, além dos conhecimentos básicos para utilizá-la".

\section{TELECENTRO: UM FIXO GEOGRÁFICO PARA A INCLUSÃO DIGITAL?}

Com a globalização, a produção de fixos e fluxos, em alguns subespaços do território brasileiro tornou-se mais densa (SANTOS 1996, [1979] 2008). O Estado tem construído e modernizado os sistemas de fluidez (estradas, portos, aeroportos), além de induzir a promoção de sistemas de fluxos de comunicação (telefones e internet, por exemplo), pois a informação e as finanças, que se valem do uso desses sistemas, tornaram-se fundamentais na dinâmica territorial, onde o capital especulativo se espraia e acirra as desigualdades regionais. Nesse sentido, autores como Benko e Lipietz, (2000), Porter (1993), permitem pensar que o processo da globalização ao se instalar nos territórios, promovem formas de inovação e competitividade.

O cidadão comum também é inserido nesse processo, seja por meio do consumo "espontâneo" de produtos e serviços, seja como mão-de-obra qualificada ou em vias de qualificação. No caso brasileiro, desde os anos de 1990 quando se inicia a expansão da internet, o governo federal passou a implementar um conjunto de políticas públicas com vistas a promover a "inclusão digital" e o acesso à internet para a população desprovida dos meios necessários ao seu uso. Dentre essas políticas destaca-se o incentivo a criação de centros públicos de acesso à internet, cuja expressão mais acabada é a criação da Política Nacional de Apoio à Inclusão Digital nas Comunidades (Telecentros.Br), por meio do Decreto N. 6.991/2009. Políticas dessa natureza, contribuíram para a estruturação de salas dotadas de equipamento de informática básico (computador, mobiliário, etc), onde, além do acesso à internet, promove-se cursos voltados a qualificação de jovens em vias de ingresso no mercado de trabalho. De acordo com o CGI.Br (2014, p. 17), telecentro é "um centro de acesso público gratuito às tecnologias de informação e comunicação".

Os telecentros estão presentes em todo território brasileiro, mas a sua maior densidade, a exemplo do que se demonstrou na Figura 1 para o caso do acesso à internet por telefone móvel de uso pessoal, está na Região Concentrada. Na Figura 2 se apresenta o número de telecentros por unidade da federação no período de 2008 a 2013.

Se por um lado, por meio da Figura 2, é possível observar que os anos de 2008 e 2009 são marcados pela expansão dos telecentros por todo território nacional, uma vez que nesse período, o governo federal lançou programas que contribuíram para a expansão no número dos telecentros instalados, quais sejam: Programa Territórios Digitais (2008); Programa Banda Larga nas Escolas (2008); Programa Nacional de Apoio à Inclusão Digital nas Comunidades - Telecentros.Br (2009). Destaque-se ainda que mesmo em 2014, após o lançamento do Programa Amazônia Conectada, cujo objetivo, de acordo com o Tribunal de Contas da União (2015), era "integrar, via fibra óptica subfluvial, o interior do estado do Amazonas", ele permanece sendo um estado de baixa densidade no que se refere a presença dos telecentros. Nesse sentido, na Região Norte, onde situa-se o Amazonas, apenas o estado do Pará foi beneficiado com a expansão no número de telecentros. Por outro lado, uma comparação breve entre as Figuras 1 e 2, demonstra que a medida que ocorreu uma maior expansão do acesso à internet por telefone celular por unidade da federação, notadamente no período de 2012 em diante, tem-se uma maior redução de telecentros instalados no território nacional. 
Figura 2. Evolução do número de Telecentros instalados por unidade da federação (2008-2013).
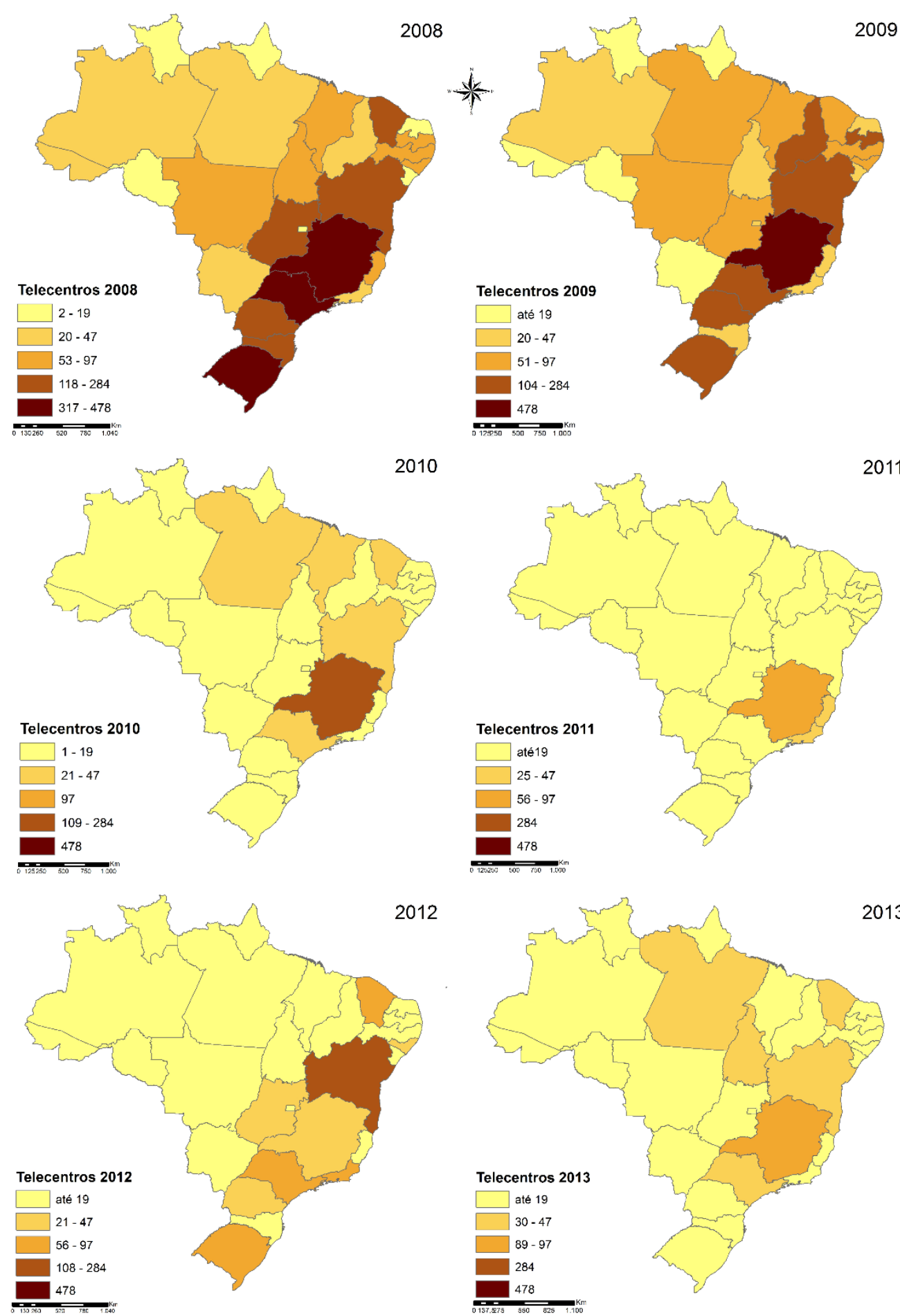

Fonte: MCTIC, 2017

Autores: D. Paz \& J. Barbosa 


\section{CONSIDERAÇÕES FINAIS}

O processo de expansão da internet, incrementado pelo uso de telefones celulares de uso pessoal, alcançou todos os estados da federação brasileira, mas seu acesso ainda não é massificado. Políticas públicas como a dos Telecentros.Br do governo federal, ao mesmo tempo que oferecem maiores possibilidades de acesso à população, concentra-se nos subespaços de maior dinâmica econômica e financeira do território nacional, reforçando velhas centralidades e desigualdades regionais.

\section{AGRADECIMENTOS}

Agradeço ao apoio recebido pela Coordenação de Aperfeiçoamento de Pessoal de Nível Superior (CAPES) pela bolsa Pós-Doutoral PNPD/CAPES, vinculada ao Programa de Pós-Graduação em Geografia Humana da Universidade Federal do Rio Grande do Norte (UFRN), a qual permitiu a realização da pesquisa que deu origem a esse artigo.

\section{REFERÊNCIAS}

ALVES, L. G. Redes de comunicação e território: a formação e a organização socioespacial da internet no Brasil. Dissertação (Mestrado em Geografia Humana) - Faculdade de Filosofia, Letras e Ciências Humanas, Universidade de São Paulo, São Paulo. 2013

BENKO, G.; Lipietz, A (Orgs). La Richesse des Régions: la nouvelle géographie sócio-économique. Paris: Puf. 2000.

BRASIL. Tribunal de Contas da União - TCU. Política pública de inclusão digital. Brasília: TCU, SeInfraAeroTelecom. 2015.

CASTELLS, M. A Sociedade em Rede. Volume I. $8^{\text {a }}$ edição revista e ampliada. São Paulo: Paz e Terra. 1999. Zahar, 2003.

A Galáxia da Internet: reflexões sobre a internet, os negócios e a sociedade. Rio de Janeiro:

Comitê Gestor da Internet no Brasil - CGI.BR. Pesquisa Sobre o Uso das Tecnologias de Informação e Comunicação no Brasil: tic centros públicos de acesso 2013. São Paulo: CGI.BR. 2014.

FUSER, B. Sociedade em Rede, Inclusão Digital e Cidadania Cultural. In: OLIVEIRA, C. T. de F; NUNES, M. V. (Orgs.). Cidadania e Cultura Digital: apropriações populares da Internet. Rio de Janeiro: E- papers, 2011. p.13-44.

GRAS, Alain. Grandeur et dépendance: sociologie des macrosystèmes techniques. Paris: Presses universitaires de France. 1993.

LATOUR, B. Jamais fomos modernos. Ensaio de antropologia simétrica. Rio de Janeiro: Editora 34. 152p. 1994.

LÉVY, P. Cibercultura. São Paulo: Editora 34. 1997.

OLIVEIRA, T. B. Território e Internet: uma análise a partir da capilaridade dos centros públicos de acesso em Alagoas. Dissertação (Mestrado em Geografia) - Instituto de Geografia, Desenvolvimento e Meio Ambiente, Universidade Federal de Alagoas - UFAL. 2016

POTER, M. A vantagem competitiva das nações. Rio de Janeiro: Campus. 1993.

SANTOS, M. A natureza do espaço: técnica, tempo, razão e emoção. ed. 4. São Paulo: EDUSP. 384p. ([1996] 2008

O Espaço dividido: os dois circuitos da economia urbana dos países subdesenvolvidos. São Paulo: EDUSP, 440p. ([1979] 2008) 
SANTOS, M; SILVEIRA, M. L. O Brasil: território e sociedade no Século XXI. Rio de Janeiro: Record. 2006

STOPER, M.; VENABLES, A. J. "O Burburinho": a força econômica da cidade. In: Diniz, C. C.; Lemos, M. B. (Orgs). Economia e Território. Belo Horizonte: UFMG. p. 21-56. 2005.

TOWSEND, A. M. (2014). Smart Cities: big data, civic hackers and the quest of new utopia. Paperback. 2014. 